\title{
CDNA-AFLP ANALYSIS REVEALS INDUCIBLE GENE EXPRESSION IN TOMATO LEAVES IN RESPONSE TO SIMULATED ACID RAIN
}

\author{
LIU, T. T. - MA, J. - LI, M. - PAN, T. F. - MA, C. L. - QIU, D. L. \\ College of Horticulture, Fujian Agriculture and Forestry University, Fuzhou 350002, China \\ *Corresponding author \\ e-mail: qiudl1970@fafu.edu.cn; phone: +86-591-8378-9379
}

(Received $21^{\text {st }}$ Jan 2019 ; accepted $27^{\text {th }}$ Feb 2019)

\begin{abstract}
Acid rain as an important environmental problem in China and a stressor may cause gene expression, and tomato is one of model plants and the most important vegetable crops in middle and south China. Tomato seedlings were exposed for 7 days to simulated acid rain (SiAR) of pH 3.0 and control (pH 5.6). The cDNA-amplified fragment length polymorphism (cDNA-AFLP) was carried out to separate differentially expressed gene fragments by using selective base pairs including 8 Mse I primers and 8 EcoR I primers, which allowed the identification and cloning of 45 differential expression transcriptderived fragments (TDFs) corresponding to SiAR, among of which 45 TDFs were successfully cloned and sequenced. 31 of them were found homologies in the NCBI, and 19 displayed homology to genes with known functions, 12 to genes with uncharacterized function. 8 TDFs played significant role in tomato resistance to acid rain-stress. Among of them, TDF5 and TDF8 were stress- inducible transcription factor. TDF4 and TDF20 played important role in resistance to SiAR stress. TDF2 was related to isoprenoid biosynthesis, TDF7 to transcription regulation, TDF11 to metabolism, TDF24 to plant development and defense. Possible roles of the 8 TDFs in the response of tomato to SiAR are also discussed. Moreover, Homologous of amino acid encoded in TDF5 and TDF8 were analyzed.

Keywords: SiAR stress, Lycopersicum esculentum, cDNA-amplified fragment length polymorphism, transcript-derived fragments
\end{abstract}

Abbreviations: SiAR: simulated acid rain, cDNA-AFLP: cDNA-amplified fragment length polymorphism, TDFs: transcript-derived fragments

\section{Introduction}

Acid rain emerged as an important environmental problem in China in the late 1970s. The frequency of acid rain has kept increasing since then. It was reported that the loss of forest ecological benefits caused by acid rain exceeded 16 billion Yuan (2.4 billion dollars) per year only in 11 provinces of south China (Feng, 2000). Previous studies have documented acid rain stress causing the leaching of leaf nutrients; changes of physiological and biochemical process; and inhibition of growth and yield of plants (Bellani et al., 1997; Chen et al., 2013; Odiyi et al., 2014; Percy et al., 1990; Qiu et al., 2002, 2005; Ramlall et al., 2015; Wang et al., 2014a, b) Acid rain as an environmental stressor may cause gene differential expression. Gene expression profiles were reported in Dimocarpus longan Lour (Zheng et al., 2017), Synechocystis sp PCC 6803 (Ohta et al., 2005) and Arabidopsis thaliana (L.) Heynh (Liu et al., 2013) in response to acid rain stress. Changes in the total protein profile of Dimocarpus longan leaves in response to simulated acid rain (SiAR) stress were documented by Pan et al. (2015).

Tomato (Lycopersicon esculentum) is one of model plants and the most important vegetable crops in middle and south China. Previous study has shown that the pigment synthesis, growth and biomass of tomato was inhibited under SiAR stress (Shaukat et al., 2008). One way to study the cellular response to acid rain on a large scale is to 
examine gene expression at the mRNA level using the PCR-based technique of cDNAamplified fragment length polymorphism (cDNA-AFLP) which has often been described as an extremely efficient method for isolation of differentially expressed genes (Baisakh et al., 2006) and is a less labor-intensive mRNA fingerprinting method for isolation of differentially expressed genes (Bachem et al., 1996). Due to its high sensitivity, it is possible to identify rare transcripts (Fukumura et al., 2003). This technique has been further improved to avoid the possibility of several transcriptderived fragment (TDFs) arising from a single gene/cDNA. Keeping the above in view, the objective of this study was to identify some of the key genes that were differentially expressed in response to SiAR.

\section{Materials and methods}

\section{Plant materials and treatments}

Tomato seeds (Lycopersicum esculentum cv. 'Fudan') were sown in plug trays with peat-based substrate with peat, vermiculite, and perlite medium (3:1:1). 7 days after emergence (DAE), each seedling was transferred to a pot containing a 3:1:1 mixture of peat, vermiculite, and perlite. The plants were kept in a net house with a natural photoperiod at Fujian Agriculture and Forestry University. Simulated acid rain was applied to plants at 42 DAE.

Sulfuric acid and nitric acid were selected for the preparation of SiAR, based on the mole ratio 1:5 of sulfuric acid to nitric acid in the precipitation of southern Fujian, China (Tang et al., 1996). Normal rain has a pH of about 5.6; it is slightly acidic because carbon dioxide $\left(\mathrm{CO}_{2}\right)$ dissolves into it forming weak carbonic acid, and the solution with $\mathrm{pH}$ value of 5.6 was regarded as the control (Qiu et al., 2005). Solution of SiAR with $\mathrm{pH} 3.0$ was also prepared. Dilution of reagent grade acid was done with distilled water and determined by Orion 828 acidity analyzer (made in America). A sprayer was used to apply the acid solutions. The spray was repeated 7 times during 7 days, at each application, leaves were thoroughly wetted. There were 9 pots in each treatment, and each treatment was repeated triple. Each replicate consisted of 9 plants. After 7 days of application, one leave from each tested plant (symptoms appeared) and control plant (Fig. 1) was sampled and immediately frozen in liquid nitrogen and then stored at $-80^{\circ} \mathrm{C}$ until use.
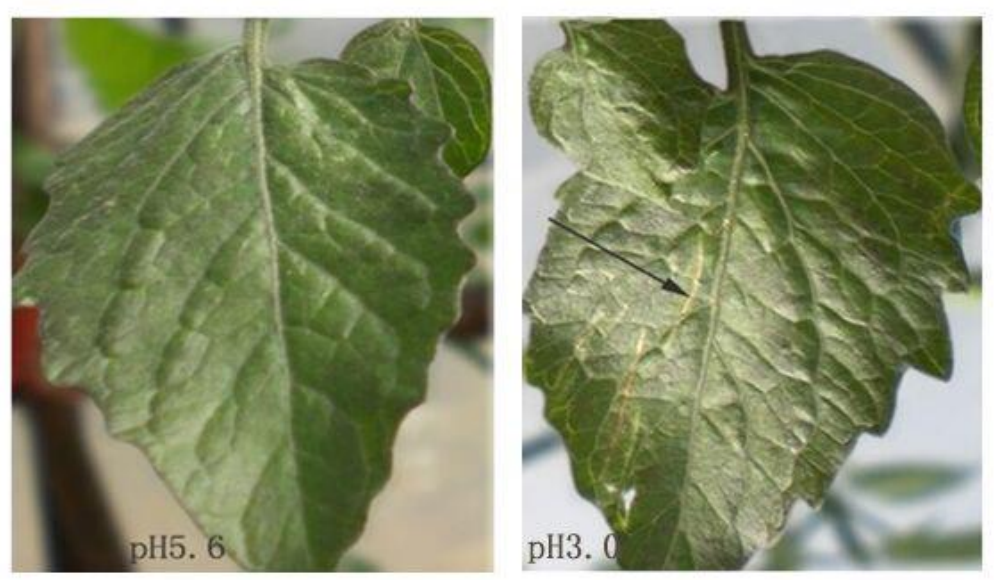

Figure 1. Tomato leaves after treated by pH 3.0 and control (pH 5.6) for 1 week 


\section{RNA preparation and cDNA synthesis}

Total RNA was isolated from each sample of frozen tissue $(0.1 \mathrm{~g})$. The frozen tissue was homogenized with $0.5 \mathrm{ml}$ of isopropyl alcohol $(1: 10$, v/v) per $1 \mathrm{ml}$ with Trizol extraction method (Invitrogen, Shanghai). The RNA concentration was determined by measuring absorbance at $260 \mathrm{~nm}$. RNA integrity was determined by running $5 \mu \mathrm{L}$ of total RNA in a $1.0 \%$ agarose gel electrophoresis. $4 \mu \mathrm{g}$ of total RNA was used initially for first strand synthesis, followed by second strand synthesis using SuperScript ${ }^{\mathrm{TM}}$ III double stranded cDNA synthesis kit (Invitrogen), according to the manufacturer's protocol.

\section{cDNA-AFLP reaction}

The cDNA-AFLP procedure was conducted as described by Bachem et al. (1998) with some modifications. Approximately $100 \mathrm{ng}$ cDNA from from each treatment was digested with EcoRI and MseI restriction enzymes in a three-step reaction at $37^{\circ} \mathrm{C}$ for $55 \mathrm{~min}, 65^{\circ} \mathrm{C}$ for $55 \mathrm{~min}$ and $80^{\circ} \mathrm{C} 10 \mathrm{~min}$, respectively. The digested products were ligated to adapters with sequences as follows: EcoRI adapter, 5' CTC GTA GAC TGC GTA CC 3', 3'CAT CTG ACG CAT GGT TAAP5'; MseI adapter, 5'GAC GAT GAG TCC TGA G 3', 3'TAC TCA GGA CTC ATP5'. The ligated products were pre-amplified with the corresponding pre-amplification primers (EcoRI: 5' GAC TGC GTA CCA ATT CA 3', MseI: 5'GAT GAG TCC TGA GTAA C 3'5). Equal amounts of pre-amplified products were amplified with primers having selective nucleotides at the 3' end (EcoRI: 5' GAC TGC GTA CCA ATT CA AC 3'; MseI: 5'GAT GAG TCC TGA GTAA C AA 3' (Table 1). Altogether 128 different primer combinations were tested. $4 \mu \mathrm{L}$ of the AFLP products were heat-denatured and resolved in a $6 \%$ denaturing polyacrylamide sequencing gel run with $1 \times$ TBE electrohphoresis buffer. The gels were silver-stained according to the Silver SequenceTM DNA Sequencing System Technical Manual.

\section{Transcript-derived fragment (TDF) isolation and re-amplification}

The bands of interest were marked, removed from the gel and soaked in $\mathrm{ddH}_{2} \mathrm{O}$ $(50 \mu \mathrm{L})$ initially at $100{ }^{\circ} \mathrm{C}$ for $15 \mathrm{~min}$ and then hydrated overnight at $4{ }^{\circ} \mathrm{C} .10 \mu \mathrm{L}$ of the aliquot was used for re-amplification in a total volume of $50 \mu \mathrm{L}$, with the same set of corresponding selective primers and the same PCR conditions as for the selective amplification except that an annealing temperature of $56{ }^{\circ} \mathrm{C}, 35$ cycles and a final 4 min extension were used. The PCR products were resolved in a $1.5 \% 1 \times$ TBE-agarose gel, and each single band corresponding to the selected TDFs was isolated, soaked to elute the DNA according to agarose gel DNA Purification Kit (TAKARA).

\section{Cloning and sequencing of TDFs}

The eluted TDFs were cloned into the plasmid $\mathrm{pMD}_{18}-\mathrm{T}$ vector (TaKaRa Biotechnology (Dalian) Co., Ltd., China) following the manufacturer's protocol. Positive clones were inoculated, and PCR positive cultures from every TDF were sequenced with an $\mathrm{ABI}$ automated sequencer.

\section{Analysis of sequences}

The sequences of the TDF were analyzed for their homology against the publicly available non-redundant genes/ESTs/Transcripts in GeneBank using the BLASTN and BLASTX algorithms. Multiple sequence alignment was performed with ClustalX2.0 
algorithm with default parameters (http://www.clustal.org/clustal2/). The phylogenetic tree was constructed using the neighbor-joining method in MEGA5.0 package (Tamura et al., 2011).

Table 1. Primers used for cDNA-AFLP amplification

\begin{tabular}{|c|c|c|}
\hline Adaptorlprimers & Primer NO. & Primer sequence \\
\hline Mse I Adapter & & $\begin{array}{c}\text { 5'GAC GAT GAG TCC TGA G 3' } \\
\text { 3'TAC TCA GGA CTC ATP5' }\end{array}$ \\
\hline Mse I Pre-amp & M00 & 5'GAT GAG TCC TGA GTAA C 3', \\
\hline \multirow[t]{8}{*}{ Mse I Sel-smp } & M0 & 5'GAT GAG TCC TGA GTAA C AA 3' \\
\hline & M1 & 5'GAT GAG TCC TGA GTAA C AC 3' \\
\hline & M2 & 5'GAT GAG TCC TGA GTAA C AG 3' \\
\hline & M3 & 5'GAT GAG TCC TGA GTAA C AT 3' \\
\hline & M4 & 5'GAT GAG TCC TGA GTAA C TA 3' \\
\hline & M5 & 5'GAT GAG TCC TGA GTAA C TC 3' \\
\hline & M6 & 5'GAT GAG TCC TGA GTAA C TG 3' \\
\hline & M7 & 5'GAT GAG TCC TGA GTAA C TT 3' \\
\hline EcoR I Adapter & & $\begin{array}{l}\text { 5' CTC GTA GAC TGC GTA CC 3', } \\
\text { 3'CAT CTG ACG CAT GGT TAAP5' }\end{array}$ \\
\hline EcoR I Pre-amp & E00 & 5’ GAC TGC GTA CCA ATT CA 3' \\
\hline \multirow[t]{8}{*}{ EcoR I Sel-smp } & E0 & 5' GAC TGC GTA CCA ATT CA AC 3' \\
\hline & E1 & 5' GAC TGC GTA CCA ATT CA AG 3' \\
\hline & E2 & 5' GAC TGC GTA CCA ATT CA CA 3' \\
\hline & E3 & 5' GAC TGC GTA CCA ATT CA CT 3' \\
\hline & E4 & 5' GAC TGC GTA CCA ATT CA CC 3' \\
\hline & E5 & 5' GAC TGC GTA CCA ATT CA CG 3' \\
\hline & E6 & 5' GAC TGC GTA CCA ATT CA GC 3' \\
\hline & E7 & 5' GAC TGC GTA CCA ATT CA GG 3' \\
\hline
\end{tabular}

\section{Results}

\section{Isolation of the TDFs responsive to SiAR stress}

A total of 128 primer combinations were used for the AFLP analysis of cDNAs from simulated acid rain-stressed leaves. cDNAs were obtained from the treatments of $\mathrm{pH} 5.6$ (Control) and pH 3.0 (Fig. 2). A total of 45 transcript-derived fragments (TDFs) were isolated from the silver-stained cDNA-AFLP gels based on their presence, and identified as inducibly expressed. Sequence alignment of these 45 cloned TDFs against NCBI databases showed that 31 had a homologous gene in the database. Due to incompleteness of tomato gene annotation and the conservation of gene families between tomato and Arabidopsis, Vitis vinifera, Populus, Ricinus communis,. Phaeosphaeria nodorum, Glycine max, Ipomoea batatas, Sorghum, Zea mays, Artemisia annua.

We used these matches of the identified inducibly expressed genes as surrogates for function annotation (Fig. 3). 14 of 45 TDFs had no annotation in the database. Fourteen of the TDFs had no similar sequences in the NCBI databases. The genes with unknown functions consist of those that have not yet been associated with a response to abiotics stress, and some of these novel genes did not match any sequence from the GenBank 
databases. In general, our results indicated that multiple defense strategies lead to an enhanced defense capacity of tomato plants to SiAR.

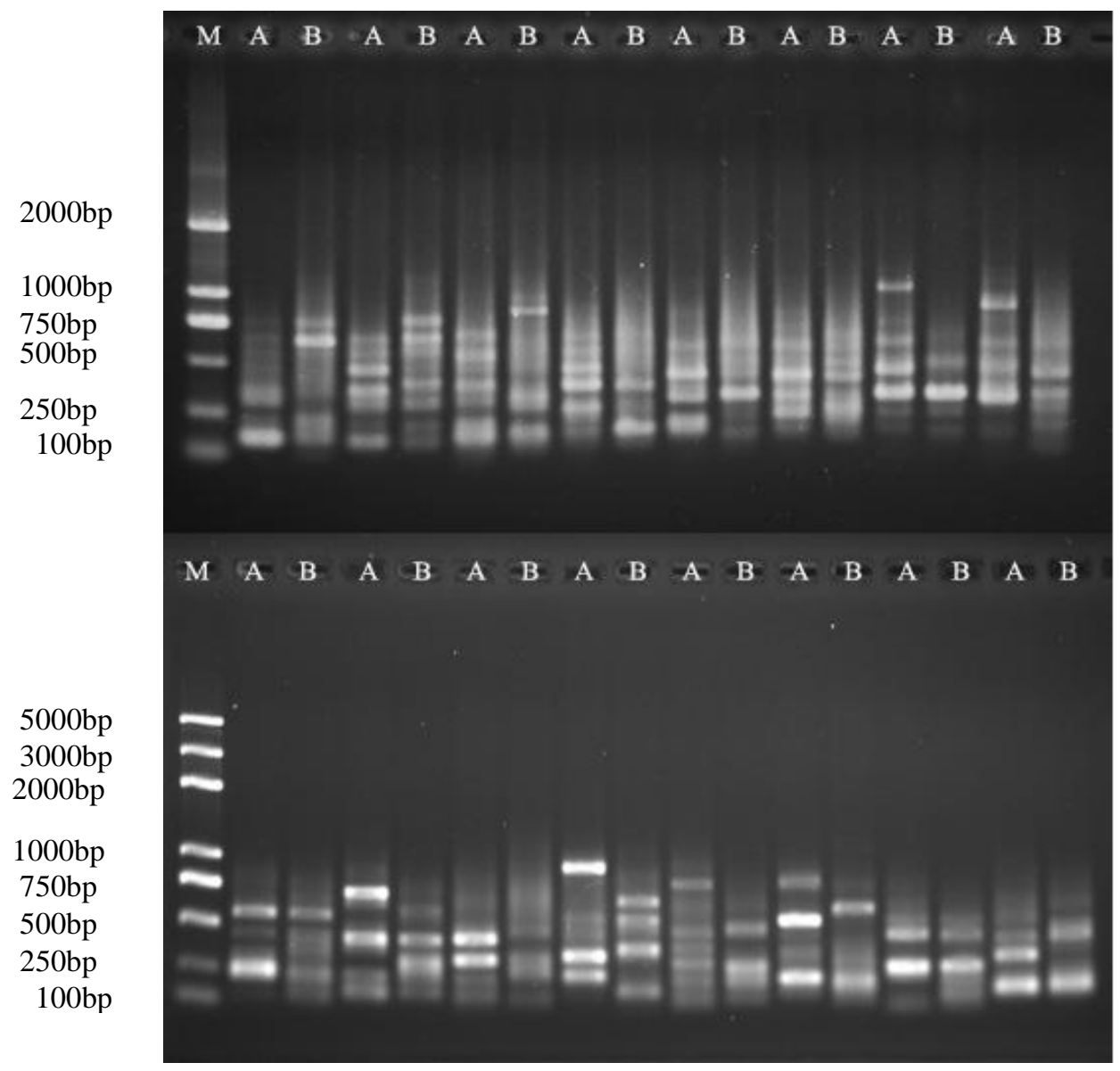

Figure 2. The result of electrophoresis of selectivity amplification products amplified with pair primers of EcoR I and Mse I (A: pH 3.0, B: pH 5.6) M: DL2000

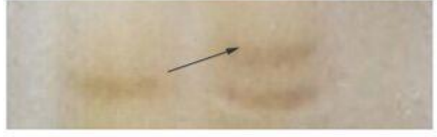

TDF24
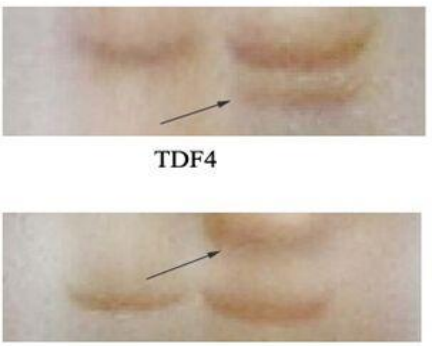

pH3.0 ${ }^{\text {TDF5 }}{ }_{\mathrm{pH} 5.6}$

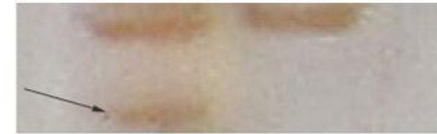

TDF 12

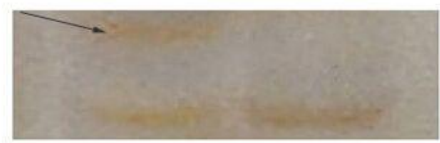

TDF17

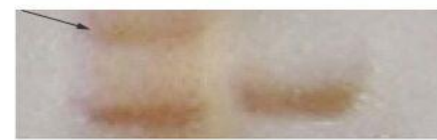

TDF3 $\mathrm{pH} 3.0$

Figure 3. Inducible expression of genes of tomato treated under SiAR and control (pH 5.6). Arrow in TDF3, TDF12, and TDF17 showed their down-regulated expression of tomato leaves in response to SiAR, while TDF4, TDF5 and TDF24 showed their up-regulated expression, respectively 


\section{Sequencing and classification of cDNA}

The expression profile was compared between $\mathrm{pH} 3.0$ and control on the basis of their either complete presence or absence (Fig. 2). 31 TDFs above 100 bp were eluted from the gel, re-amplified, and sequenced (Fig. 4 and Fig. Al in the Appendix).

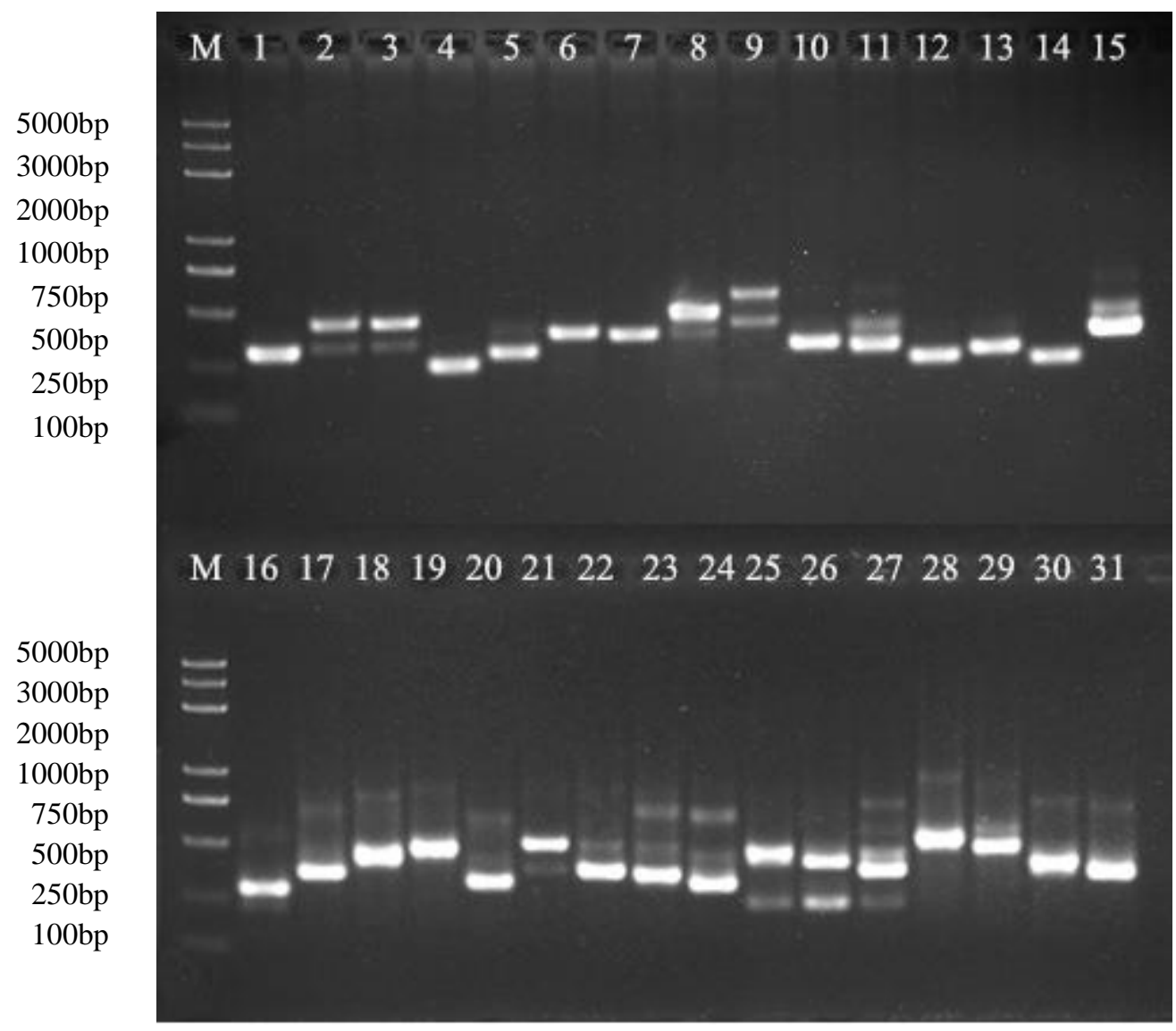

Figure 4. Agarose gel of re-amplified TDFs from tomato in response to SiAR. M: $2000 \mathrm{bp}$ marker;1-31 showing TDF1-TDF31, respectively

The TDFs with known or putative function were submitted to the NCBI database and are presented in Table 2 with GenBank Accession numbers. The up- and down-regulated genes are also categorized into these functional groups (Table 2). Figure 5 shows the percentages of acid rain induced genes assigned to different functional categories. Comparison of the homologies of these sequences and those in the database suggested that most of them (about 68.9\%) had homology with genes involved in functions like UBA and UBX domain-containing protein (12.9\%, TDF1, TDF6, TDF9, TDF19), Inositol-1, 4, 5 -triphosphate-5-phosphatase (3.2\%, TDF15), Inositol polyphosphate 5-phosphatase $(3.2 \%$, TDF26), Tobamovirus multiplication protein (3.2\%, TDF13), Hypothetical protein (9.7\%, TDF11, TDF16, TDF28), RING/U-box superfamily protein (3.2\%, TDF7), Stress transcription factor A-6b (6.5\%, TDF5, TDF8), Receptor protein (3.2\%, TDF27), Plastidic aldolase (3.2\%, TDF30), Phenylalanine ammonia-lyase (3.2\%, TDF24), Solanesyl diphosphate synthase $(3.2 \%$, TDF2), Peroxidase (6.5\%, TDF4, TDF20). 
Table 2. Transcript-derived fragments (TDFs) from tomato in response to simulated acid rain

\begin{tabular}{|c|c|c|c|c|c|c|c|c|}
\hline $\begin{array}{c}\text { TDF } \\
\text { No. }\end{array}$ & $\begin{array}{c}\text { TDF } \\
\text { size (bp) }\end{array}$ & $\begin{array}{l}\text { Sequence } \\
\text { name }\end{array}$ & $\begin{array}{c}\text { GeneBank } \\
\text { accession no. }\end{array}$ & Annotation & Similarity & E-value & Organism & Expression $^{\mathbf{a}}$ \\
\hline \multicolumn{9}{|c|}{ UBA and UBX domain-containing protein } \\
\hline 1 & 175 & M0E0-1 & AK327205.1 & $\begin{array}{c}\text { cDNA, clone: LEFL2024I13, } \\
\text { HTC in fruit }\end{array}$ & $74 \%$ & $4 \mathrm{e}-15$ & $\begin{array}{c}\text { Solanum } \\
\text { lycopersicum }\end{array}$ & - \\
\hline 6 & 220 & M5E2 & AK321642.1 & \begin{tabular}{c|} 
cDNA, clone: \\
LEFL1027AF01, HTC in leaf \\
\end{tabular} & $87 \%$ & $1 e-68$ & $\begin{array}{c}\text { Solanum } \\
\text { lycopersicum }\end{array}$ & - \\
\hline 9 & 264 & M6E2-3 & AK247661.1 & \begin{tabular}{c|} 
cDNA, clone: \\
LEFL1085DG07, HTC in leaf \\
\end{tabular} & $100 \%$ & $1 \mathrm{e}-05$ & $\begin{array}{c}\text { Solanum } \\
\text { lycopersicum }\end{array}$ & - \\
\hline 19 & 387 & M5E3 & AK247676.1 & $\begin{array}{c}\text { cDNA, clone: } \\
\text { LEFL1089BC10, HTC in leaf }\end{array}$ & $79 \%$ & $4 \mathrm{e}-90$ & $\begin{array}{c}\text { Solanum } \\
\text { lycopersicum }\end{array}$ & - \\
\hline \multicolumn{9}{|c|}{ Inositol-1,4,5-triphosphate-5-phosphatase } \\
\hline 15 & 170 & M5E0 & EU159402.1 & $\begin{array}{c}\text { Inositol-1,4,5-triphosphate-5- } \\
\text { phosphatase (5PT1) mRNA, } \\
\text { complete cds }\end{array}$ & $100 \%$ & $4 \mathrm{e}-22$ & $\begin{array}{c}\text { Solanum } \\
\text { lycopersicum }\end{array}$ & - \\
\hline \multicolumn{9}{|c|}{ Inositol polyphosphate 5-phosphatase } \\
\hline 26 & 225 & M7E2-3 & XM_010326266.2 & \begin{tabular}{|c} 
Putative inositol \\
polyphosphate 5-phosphatase \\
At5P2
\end{tabular} & $81 \%$ & $4 \mathrm{e}-18$ & $\begin{array}{c}\text { Solanum } \\
\text { lycopersicum }\end{array}$ & - \\
\hline \multicolumn{9}{|c|}{ Tobamovirus multiplication protein } \\
\hline 13 & 360 & M2E3 & XP_010318614.2 & $\begin{array}{l}\text { Tobamovirus multiplication } \\
\text { protein } 1 \\
\end{array}$ & $100 \%$ & 0 & $\begin{array}{c}\text { Populus } \\
\text { trichocarpa }\end{array}$ & - \\
\hline \multicolumn{9}{|c|}{ Hypothetical protein } \\
\hline 11 & 267 & M0E3 & XM_001793885.1 & $\begin{array}{l}\text { SN15 hypothetical protein } \\
\text { partial mRNA }\end{array}$ & $72 \%$ & $1 e-26$ & \begin{tabular}{|c|} 
Phaeosphaeria \\
nodorum
\end{tabular} & + \\
\hline 16 & 301 & M2E2 & XM_002264451.1 & \begin{tabular}{|c|} 
Predicted: hypothetical protein \\
LOC100244254, mRNA
\end{tabular} & $77 \%$ & $3 e-59$ & Vitis vinifera & - \\
\hline 28 & 350 & M5E3 & XM_002513344.1 & Cyclin-L2, putative, mRNA & $85 \%$ & $2 \mathrm{e}-30$ & $\begin{array}{l}\text { Ricinus } \\
\text { communis }\end{array}$ & - \\
\hline \multicolumn{9}{|c|}{ RING/U-box superfamily protein } \\
\hline 7 & 338 & M6E2-1 & NM_123389.3 & $\begin{array}{l}\text { Zinc finger (C3HC4-type } \\
\text { RING finger) family protein } \\
\text { (AT5G40250) mRNA, } \\
\text { complete cds }\end{array}$ & $73 \%$ & $4 e-19$ & $\begin{array}{l}\text { Arabidopsis } \\
\text { thaliana }\end{array}$ & + \\
\hline \multicolumn{9}{|c|}{ Stress transcription factor $\mathbf{A - 6 b}$} \\
\hline $8^{\mathrm{b}}$ & 290 & M6E2-2 & XM_002517024.1 & $\begin{array}{l}\text { Heat shock factor protein } \\
\text { HSF30, putative, mRNA }\end{array}$ & $76 \%$ & $1 e-37$ & $\begin{array}{l}\text { Ricinus } \\
\text { communis }\end{array}$ & + \\
\hline $5^{\mathrm{b}}$ & 202 & M5E0-2 & CU227021.1 & $\begin{array}{l}\text { EST from severe drought- } \\
\text { stressed leaves }\end{array}$ & $76 \%$ & $2 \mathrm{e}-48$ & Populus & + \\
\hline \multicolumn{9}{|c|}{ Receptor protein } \\
\hline 27 & 344 & M3E3 & AF401036.1 & $\begin{array}{l}\text { Receptor-like protein 9DC } \\
\text { gene, complete cds }\end{array}$ & $78 \%$ & $3 e-66$ & \begin{tabular}{|c|} 
Lycopersicon \\
pimpinellifolium
\end{tabular} & + \\
\hline \multicolumn{9}{|c|}{ Plastidic aldolase } \\
\hline 30 & 365 & M7E3-1 & AB027001.1 & $\begin{array}{l}\text { mRNA for plastidic aldolase } \\
\text { NPALDP1, complete cds }\end{array}$ & $80 \%$ & $5 e-50$ & $\begin{array}{l}\text { Nicotiana } \\
\text { paniculata }\end{array}$ & + \\
\hline \multicolumn{9}{|c|}{ Phenylalanine ammonia-lyase } \\
\hline 24 & 298 & M7E2-1 & M90692.1 & $\begin{array}{c}\text { Phenylalanine ammonia-lyase } \\
\text { (PAL5) gene, complete cds }\end{array}$ & $95 \%$ & $8 \mathrm{e}-129$ & $\begin{array}{l}\text { Lycopersicon } \\
\text { esculentum }\end{array}$ & + \\
\hline \multicolumn{9}{|c|}{ Solanesyl diphosphate synthase } \\
\hline 2 & 338 & MOE0-2 & DQ889204.1 & $\begin{array}{l}\text { Solanesyl diphosphate } \\
\text { synthase (SppS) gene, } \\
\text { complete cds }\end{array}$ & $100 \%$ & $1 \mathrm{e}-171$ & $\begin{array}{l}\text { Lycopersicon } \\
\text { esculentum }\end{array}$ & + \\
\hline \multicolumn{9}{|c|}{ Peroxidase } \\
\hline 4 & 341 & M5E0-1 & BAG09369.1 & Peroxisomal acyl-CoA oxidase & $87 \%$ & $3 e-14$ & Glycine $\max$ & + \\
\hline 20 & 206 & M1E0-2 & AY206413.1 & $\begin{array}{l}\text { Anionic peroxidase swpb2 } \\
\text { mRNA, complete cds }\end{array}$ & $78 \%$ & $3 e-30$ & $\begin{array}{l}\text { Ipomoea } \\
\text { batatas }\end{array}$ & + \\
\hline
\end{tabular}




\begin{tabular}{|c|c|c|c|c|c|c|c|c|}
\hline \multicolumn{9}{|c|}{ Uncharacterized } \\
\hline 14 & 154 & M1E0-1 & HG975443.1 & $\begin{array}{l}\text { Chromosome ch04, complete } \\
\text { genome }\end{array}$ & $100 \%$ & $2 \mathrm{e}-71$ & $\begin{array}{c}\text { Solanum } \\
\text { lycopersicum }\end{array}$ & - \\
\hline 12 & 247 & M1E1 & AC239572.2 & $\begin{array}{l}\text { Strain Heinz } 1706 \text { clone hba- } \\
\text { 139d12, complete sequence }\end{array}$ & $82 \%$ & $5 e-61$ & $\begin{array}{c}\text { Solanum } \\
\text { lycopersicum }\end{array}$ & - \\
\hline 17 & 258 & M3E2 & AC212438.2 & $\begin{array}{l}\text { Chromosome } 11 \text { clone } \\
\text { C11HBa0168B23, complete } \\
\text { sequence }\end{array}$ & $100 \%$ & $2 \mathrm{e}-128$ & $\begin{array}{c}\text { Solanum } \\
\text { lycopersicum }\end{array}$ & - \\
\hline 18 & 211 & M4E3 & CU468016.3 & $\begin{array}{l}\text { DNA sequence from clone } \\
\text { LE_HBa-187P23, complete } \\
\text { sequence }\end{array}$ & $86 \%$ & $6 e-65$ & $\begin{array}{c}\text { Solanum } \\
\text { lycopersicum }\end{array}$ & - \\
\hline 21 & 274 & M7E0 & CU457804.4 & $\begin{array}{l}\text { DNA sequence from clone } \\
\text { SL_MboI-121C14 on } \\
\text { chromosome 4, complete } \\
\text { sequence }\end{array}$ & $85 \%$ & $7 e-79$ & $\begin{array}{c}\text { Solanum } \\
\text { lycopersicum }\end{array}$ & - \\
\hline 22 & 292 & M1E2 & EU180574.1 & $\begin{array}{c}\text { Clone BAC } \\
\text { C09HBa0179M08, complete } \\
\text { sequence }\end{array}$ & $72 \%$ & $3 e-13$ & $\begin{array}{c}\text { Solanum } \\
\text { lycopersicum }\end{array}$ & - \\
\hline 23 & 281 & M2E2 & CU326380.7 & $\begin{array}{l}\text { DNA sequence from clone } \\
\text { LE_HBa-75M11, complete } \\
\text { sequence }\end{array}$ & $86 \%$ & $7 e-85$ & $\begin{array}{c}\text { Solanum } \\
\text { lycopersicum }\end{array}$ & - \\
\hline 25 & 310 & M7E2-2 & AP011551.1 & $\begin{array}{l}\text { DNA, chromosome } 8 \text {, clone: } \\
\text { C08SLe0087H10, complete } \\
\text { sequence }\end{array}$ & $80 \%$ & $9 e-40$ & $\begin{array}{c}\text { Solanum } \\
\text { lycopersicum }\end{array}$ & - \\
\hline 31 & 193 & M7E3-2 & AP011553.1 & $\begin{array}{l}\text { DNA, chromosome } 8 \text {, clone: } \\
\text { C08SLm0050D24, complete } \\
\text { sequence }\end{array}$ & $75 \%$ & $1 \mathrm{e}-04$ & $\begin{array}{c}\text { Solanum } \\
\text { lycopersicum }\end{array}$ & - \\
\hline 29 & 169 & M6E3 & AC239435.2 & $\begin{array}{c}\text { Strain Heinz } 1706 \\
\text { chromosome } 1 \text { clone hba- } \\
\text { 305j13 map } 1 \text {, complete } \\
\text { sequence }\end{array}$ & $89 \%$ & $3 e-49$ & $\begin{array}{c}\text { Solanum } \\
\text { lycopersicum }\end{array}$ & 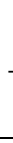 \\
\hline 10 & 212 & M6E2-4 & CU302232.4 & $\begin{array}{l}\text { DNA sequence from clone } \\
\text { LE_HBa-29F16 on } \\
\text { chromosome 4, complete } \\
\text { sequence }\end{array}$ & $74 \%$ & $4 e-23$ & $\begin{array}{c}\text { Solanum } \\
\text { lycopersicum }\end{array}$ & - \\
\hline 3 & 164 & MOE0-3 & AP010799.1 & $\begin{array}{l}\text { DNA, chromosome } 8 \text {, clone: } \\
\text { C08HBa0112M02, complete } \\
\text { sequence }\end{array}$ & $88 \%$ & $2 \mathrm{e}-47$ & $\begin{array}{c}\text { Solanum } \\
\text { lycopersicum }\end{array}$ & 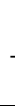 \\
\hline
\end{tabular}

a: The TDF up-regulated under SiAR stress were indicated with plus (+), while the TDFs down-regulated were indicated with minus (-); b: TDFs selected for homologous analysis of amino acid
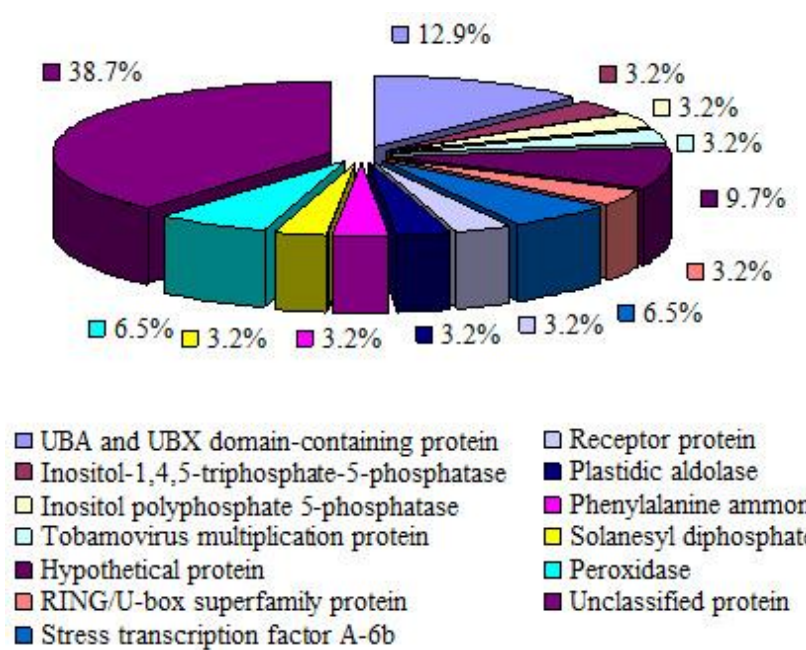

$\square$ Receptor protein

- Plastidic aldolase

$\square$ Phenylalanine ammonia-lyase

$\square$ Solanesyl diphosphate synthase

$\square$ Peroxidase

$\square$ Unclassified protein

Figure 5. Distribution of inducibly expressed genes of tomato under SiAR stress. A total of 31 TDFs were grouped into 13 functional categories. The percentages of gene transcripts in each group are listed 


\section{The homologous analysis of TDF5 and TDF8}

Multiple sequence alignment revealed that the predicted nucleotide sequence of TDF5 displayed homology with other species (Fig. 6), including Populus (0.09935), Vitis vinifera (0.21868), Ricinus communis (0.08232), Daucus carota (0.24211), Arabidopsis (0.23250), Lycopersicum esculentum (0.00804), Soybean (0.29692) (Fig. 7), while TDF8 had homology with Lycopersicum esculentum (0.12116), Vitis vinifera (0.13402), Artemisia aпnиa (0.71636), Sorghum (0.05429), Zea mays (0.08618), Ricinus communis (0.101665), Populus (0.04893), Arabidopsis (0.22726) (Figs. 8 and 9).

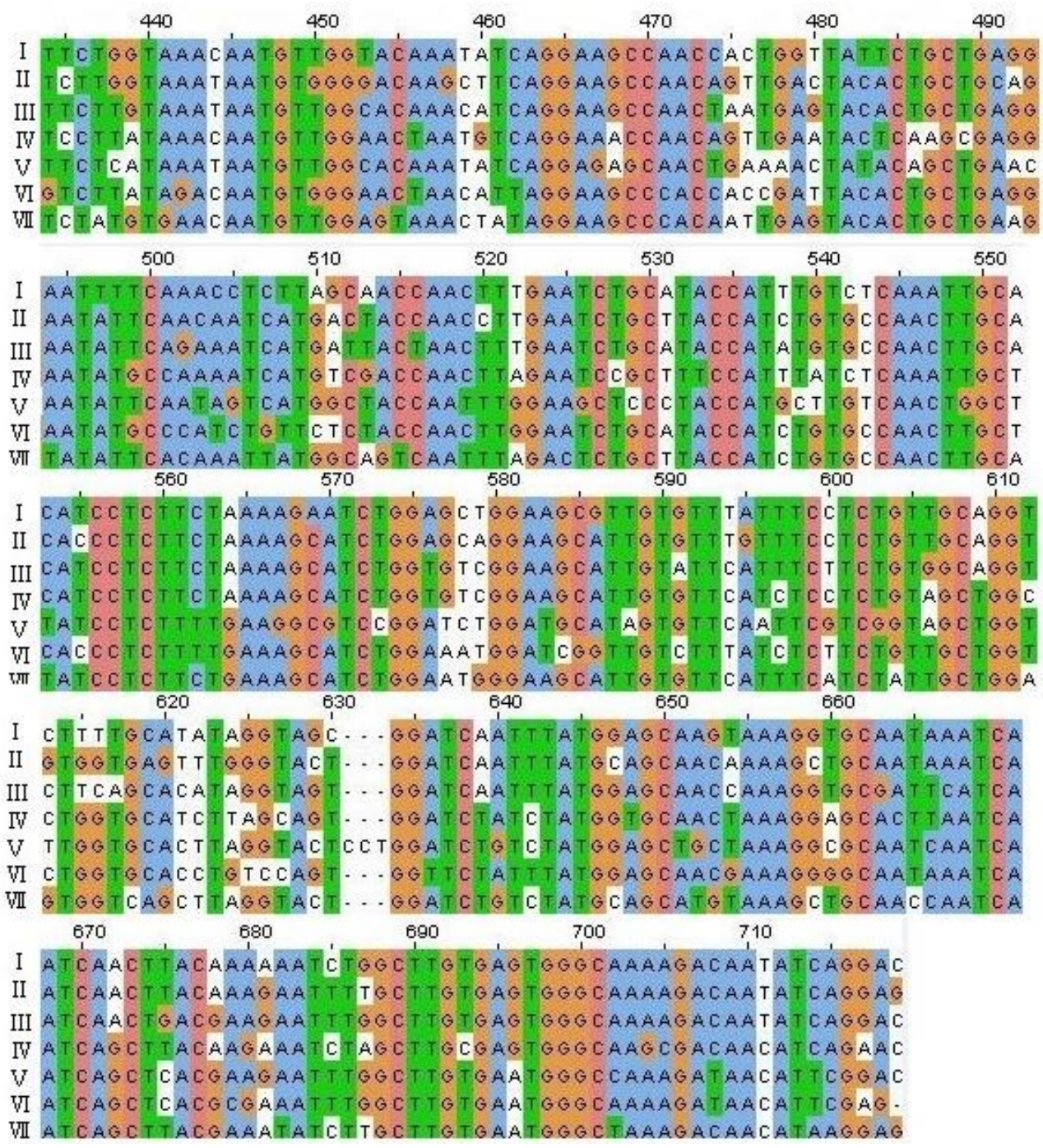

Figure 6. Homologous analysis of TDF5

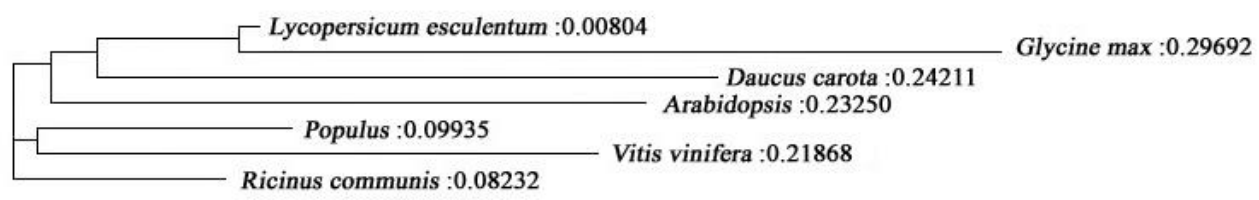

Figure 7. Homologous tree of TDF5 


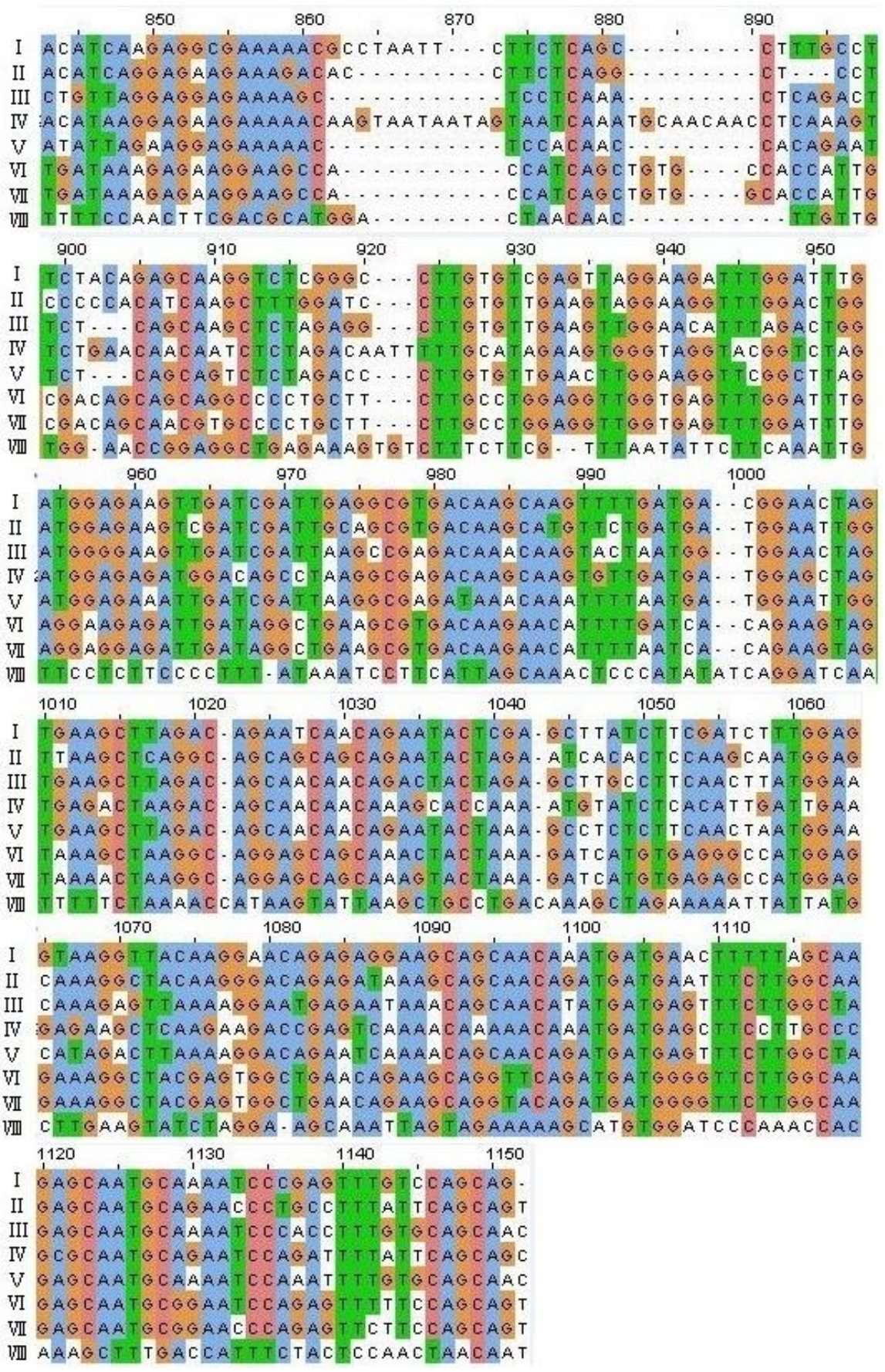

Figure 8. Homologous analysis of TDF8. I: Lycopersicum esculentum; II: Vitis vinifera; III: Populus; IV: Arabidopsis; V: Ricinus communis; VI: Sorghum; VII: Zea mays; VIII: Artemisia annua

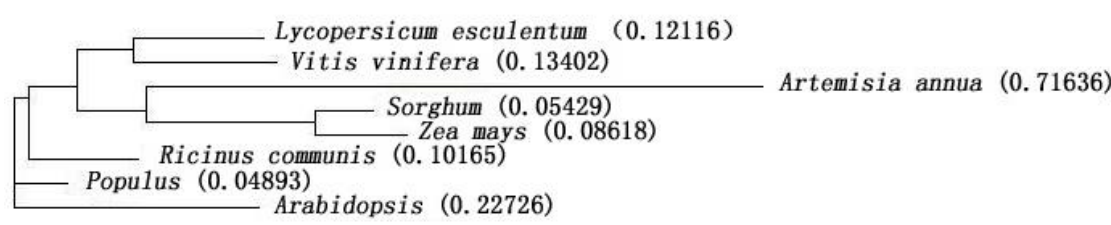

Figure 9. Homologous tree of TDF8 


\section{Discussion}

The biological functions of identified SiAR-responsive genes were categorized according to transcriptional levels. The putative functions of the TDFs that were differentially expressed in SiAR treatments were analyzed. The largest categories with ascribed function are stress-related genes $(68.9 \%)$ involved in functions like UBA and UBX domain-containing protein (12.99\%), peroxidase (6.5\%), inositol-1, 4, 5 triphosphate-5-phosphatase $(3.2 \%)$, inositol polyphosphate 5-phosphatase (3.2\%), tobamovirus multiplication protein $(3.2 \%)$, hypothetical protein $(9.7 \%)$, RING/U-box superfamily protein $(3.2 \%)$, stress transcription factor A-6b $(6.5 \%)$, receptor protein (3.2\%), plastidic aldolase (3.2\%), phenylalanine ammonia-lyase (3.2\%), solanesyl diphosphate synthase (3.2\%). Among of them, the expression of TDFs in tomato leaves under SiAR stress were up-regulated, including TDF2, TDF4, TDF5, TDF7, TDF8, TDF11, TDF20, TDF24, TDF27, TDF30. The results of the differentially regulated TDFs reported to have direct or indirect relation with simulated acid rain stress response are discussed below.

\section{Responses of isoprenoid biosynthetic genes to simulated acid rain}

As a part of the plant defense machinery, isoprenoids are employed, among others, to protect plant cells from abiotic stress (Munne-Bosch et al., 2003). TDF2 showed high similarity with the enzymatic proteins in the pathway of isoprenoid biosynthesis, which were down-regulated in the seedlings subjected to simulated acid rain stress. Plastoquinone is regulated by solanesyl diphosphatesynthase (SPPS) (Phatthiya et al., 2007). Simulated acid rain stress affecting phosphorylation of the chloroplast of longan leaves was documented by Qiu et al. (2002). These indicated that simulated acid rain stress influenced photophorylation by the way of inhibiting isopretenoid production. The same result was documented that isoprenoids in Sitka spruce are induced after mechanical wounding due to increased transcription or enzymatic activities of genes and enzymes participating in the biosynthetic pathways of these metabolites (McKay et al., 2006).

\section{Phenylalanin ammonia-lyase}

TDF24 showed similarity with phenylalanin ammonia-lyase (PAL-5) gene. Phenylalanine ammonia-lyase catalyzes the conversion of L-phenylalanine intotranscinnamate, the initial committed step of the multi-branched phenylpropanoid pathway in higher plants. This is a key biochemical reaction in both plant development and defense. In tomato, at least five different classes of PAL genes were isolated. PAL 5 was distinctly (5-6-fold) more common. TDF24 was up-regulated after simulated acid rain stress. This type of PAL gene sequence also was found to be strongly expressed and differentially regulated in response to changes in light or wounding or to infection by a plant pathogen (Chang et al., 2008).

\section{Glycosyltransferase}

Glycosyltransferases, which catalyze the transfer of a sugar residue from an activated donor to an acceptor molecule, are found in all living organisms. Since plants, in contrast to animals, are sessile organisms and cannot move away from adverse environmental conditions they need to adapt themselves to environmental stresses 
(Wang et al., 2015). TDF11 expressed in tomato which evolved distinct mechanisms by which tolerance against acid rain can be achieved.

\section{Zinc finger protein kinase}

About two to three-fold increase in the expression of the cDNA (TDF7) encoding a cys $2 /$ his 2 zinc finger protein kinase conferred tolerance to simulated acid rain stress in tomato. Over-expression of the members of zinc finger protein kinase family was also documented during salt tolerance in transgenic Arabidopsis and tobacco (Mukhopadhyay et al., 2004; Ragueh et al., 1989; Sakamoto et al., 2004). Stressresponsive zinc finger gene playing a role was reported in drought tolerance in petunia (Sugano et al., 2003).

\section{Peroxisomal acyl-CoA oxidase}

The peroxisomal acyl-CoA oxidase family plays an essential role in lipid metabolism by catalyzing the conversion of acyl-CoA into trans-2-enoyl-CoA during fatty acid betaoxidation (Fabi et al., 2010). The TDFs including TDF4, TDF5, TDF8, TDF20, TDF27 and TDF30 were differentially regulated under simulated acid rain, indicating that these proteins may take part in the mechanism of anti-SiAR and play important role in the resistance to the stress.

\section{Unknown genes}

The large group of signal transduction, transcription regulation-related protein, stress- inducible proteins $(68.9 \%$ of the 31 known or predicted genes) suggested that tomato can rapidly initiate the regulatory system and control the expression of simulated acid rain resistance-related genes. Also, many unknown genes, $31.1 \%$ of the total, had not been reported and consist of those that have not yet been associated with a response to abiotics stress, and some of these novel genes did not match any sequence from the GenBank databases, and might play important roles in the response of tomato to SiAR, indicating that multiple defense strategies lead to an enhanced SiAR defense capacity in tomato.

This work helped us to identify the responsive transcripts expressed under SiAR. Most of them are related with their previously studied genes involved in stress responses such as abiotic and biotic factors. The induction of these genes under SiAR suggests their function in possible regulation of acid rain adaptation in tomato. This is suggested by the association of most of the cDNAs with genes involved in biosynthesis of isoprenoid and phenylpropanoid, zinc finger protein kinase family, and lipid metabolism. Knowledge of acid rain tolerance can be enriched by studying the genes involved in the interconnected aspects like preventing or controlling the damage caused by acid rain, establishing the homeostatic environment in the stressful environment and resuming the normal growth at the reduced rate.

TDFs identified in our work revealed the genes related to lipid metabolism and biosynthesis of anti-oxidants of isoprenoid and phenylpropanoid, zinc finger protein kinase conferred tolerance to simulated acid rain stress in tomato which help in establishing homeostatic environment. However, some of the TDFs in the study did not show homology with any other known genes, indicating that further work is necessary to elucidate the mechanism of acid rain and biological roles of these genes in seedlings 
of tomato. This progress will bring to the research community a huge wealth of information on novel acid rain-related genes.

\section{Conclusions}

Among of which 31 TDFs in leaves of tomato under simulated acid rain stress were cloned and sequenced. TDF5 and TDF8 were stress- inducible proteins. TDF4 and TDF20 played important role in resistance to SiAR stress. TDF2 was related to isoprenoid biosynthesis, TDF7 to transcription regulation, TDF11 to metabolism, TDF24 to plant development and defense. These 8 TDFs may play significant role in tomato resistance to acid rain-stress. Further study is necessary to elucidate the roles of these genes in tomato under SiAR stress.

Acknowledgments. This work was supported by the National Natural Science Grant of China (Award no. 30400061), Natural Science Foundation of Fujian Province, China (2011J01082) and Special fund for science and technology innovation of FAFU (CXZX2016107).

Conflict of interests: The authors declare that they have no conflict of interests.

\section{REFERENCES}

[1] Bachem, C. W. B., VanderHoeven, R. S., DeBrujin, S. M., Vreugdenhil, D., Zabeau, M., Visser, R. G. F. (1996): Visualization of differential gene expression using a novel method of RNA fingerprinting based on AFLP: analysis of gene expression during potato tuber development. - Plant Journal 9: 745-753.

[2] Bachem, C. W. B., Oomen, R. J. F. J., Visser, R. G. E. (1998): Transcript imaging with cDNA-AFLP: a step-by-step protocol. - Plant Molecular Biology Reporter 16: 157-173.

[3] Baisakh, N., Subudhi, P. K., Parami, N. P. (2006): cDNA-AFLP analysis reveals differential gene expression in response to salt stress in a halophyte Spartina alterniflora Loise. - Plant Science 170: 1141-1149.

[4] Bellani, L. M., Rinallo, C., Muccifora, S., Gori, P. (1997): Effects of simulated acid rain on pollen physiology and ultrastructure in the apple. - Environmental Pollution 95: 357362.

[5] Chang, A., Lim, M. H., Lee, S. W., Robb, E. J., Nazar, R. N. (2008): Tomato Phenylalanine Ammonia-lyase gene family, highly redundant but strongly underutilized. - Journal of Biological Chemistry 283: 33591-33601.

[6] Chen, J., Wang, W. H., Liu, T., Wu, F. H., Zheng, H. L. (2013): Photosynthetic and antioxidant responses of Liquidambar formosana and Schima superba seedlings to sulfuric-rich and nitric-rich simulated acid rain. - Plant Physiology and Biochemistry 64: 41-51.

[7] Fabi, J. P., Mendes, L. R. B. C., Lajolo,.M., Nascimento, J. R. O. (2010): Transcript profiling of papaya fruit reveals differentially expressed genes associated with fruit ripening. - Plant Science 179: 225-233.

[8] Feng, Z. W. (2008): Ecological effects and control strategies of acid deposition on ecosystems in China. - Yunnan Environmental Science 19: 1-6.

[9] Fukumura, R., Takahashi, H., Saito, T., Tsutsumi, Y., Fujimori, A., Sato, S., Tatsumi, K., Araki, R., Abe, M. (2003): A sensitive transcriptome analysis method that can detect unknown transcripts. - Nucleic Acids Research 31: e94.

[10] Liu, T. W., Niu, L., Fu, B., Chen, J., Wu, F. H., Chen, J., Wang, W. H., Hu, W. J., He, J. X., Zheng, H. L. (2013): A transcriptomic study reveals differentially expressed genes 
and pathways respond to simulated acid rain in Arabidopsis thaliana. - Genome 56: 4960.

[11] McKay, S., K. A., Toudefallah, M., Martin, D. M., Alfaro, R., King, J., Bohlmann, J., Plant, A. L. (2006): Wound-induced terpene synthase gene expression in Sitka spruce that exhibit resistance or susceptibility to attack by the white pine weevil. - Plant Physiology 140: 1009-1021.

[12] Mukhopadhyay, A., Vij, S., Tyagi, A. K. (2004): Overexpression of a zinc-finger protein gene from rice confers tolerance to cold, dehydration, and salt stress in transgenic tobacco. - Proceedings of the National Academy of Science of United States of America 101: 6309-6314.

[13] Munne-Bosch, S., Alegre, L. (2003): Drought-induced changes in the redox state of atocopherol, ascorbate, and the diterpene carnosic acid in chloroplasts of Labiatae species differing in carnosic acid content. - Plant Physiology131: 1816-1825.

[14] Odiy, B. O., Bamidele, J. F. (2014): Effects of simulated acid rain on growth and yield of cassava Manihot esculenta (Crantz). - Journal of Agricultural Science 6: 96-101.

[15] Ohta, H., Shibata, Y., Haseyama, Y., Yoshino, Y., Suzuki, T., Kagasawa, T., Kamei, A., Ikeuchi, M., Enami, I. (2005): Identification of genes expressed in response to acid stress in Synechocystis sp. PCC 6803 using DNA microarrays. - Photosynthesis Research 84: 225-230.

[16] Pan, T. F., Li, Y. Y., Ma, C. L., Qiu, D. L. (2015): Calcium affecting protein expression in long an undersimulated acid rain stress. - Environmental Science and Pollution Research 22: 12215-12223.

[17] Percy, K. E., Baker, E. A. (1990): Effects of simulated acid rain on epicuticular wax production, morphology, chemical composition and on cuticular membrane thickness in two clones of Sitkaspruce [Picea sitchensis (Bong.) Carr.]. - New Phytologist 116: 9-87.

[18] Phatthiya, A., Takahashi, S., Chareonthiphakorn, N., Koyama, T., Wititsuwannakul, D., Wititsuwannakul, R. (2007): Cloning and expression of the gene encoding solanesyl diphosphate synthase from Hevea brasiliensis. - Plant Science 172: 824-831.

[19] Qiu, D. L., Liu, X. H. (2002): Effects of simulated acid rain on photosynthetic function and calcium regulation. - Chinese Journal of Applied Ecology 3: 1072-1076.

[20] Qiu, D. L., Liu, X. H., Guo, S. Z. (2005): Effects of simulated acid rain on fertility of litchi. - Journal of Environmental Sciences 17: 1034-1037.

[21] Ragueh, F., Fescur, N., Roby, D., Marco, Y. (1989): Gene expression in Nicotiana tabacum in response to compatible and incompatible isolates of Pseudomonas solanacearum. - Physiological and Molecular Plant Pathology 35: 23-33.

[22] Ramlall, C., Varghese, B., Ramdhani, S., Pammenter, N. W., Bhatt, A., Berjak, P. (2015): Effects of simulated acid rain on germination, seedling growth and oxidative metabolism of recalcitrant-seeded Trichilia dregeana grown in its natural seed bank. - Physiologia Plantarum 153: 149-160.

[23] Sakamoto, H., Maruyama, K., Sakuma, Y., Meshi, T., Iwabuch, M., Shinozaki, K., Yamaguchi-Shinozaki, K. (2004): Arabidopsis cys2/his2-type zincfinger proteins function as transcription repressors under drought, cold, and high salinity conditions. Plant Physiology 136: 2734-2746.

[24] Shaukat, S. S., Khan, M. A. (2008): Growth and physiological responses of tomato (Lycopersicon esculentum Mill.) to simulated acid rain. - Pakistan Journal of Botany 40: 2427-2435.

[25] Sugano, S., Kaminaka, H., Rybka, Z., Catala, R., Salina, J. (2003): Stress-responsive zinc finger gene ZPT2-3 plays a role in drought tolerance in petunia. - Plant Journal 36: 83041.

[26] Tamura, K., Peterson, D., Peterson, N., Stecher, G., Nei, M., Kumar, S. (2011): Mega5: Molecular evolutionary genetics analysis using maximum likelihood, evolutionary distance, and maximum parsimony methods. - Molecular Biology and Evolution 28: 2731-2739. 
[27] Tang, D. G., Wang, W., Pang, Y. B., Liu, H. J., Wang, S. S., Wang, R. M. (1996): Contribution of NOx to acid rain in the area of south Fujian province. - Research of Environmental Science 9: 38-40.

[28] Wang, L. H., Wang, W., Zhou, Q., Huang, X. H. (2014a): Combined effects of lanthanum (III) chloride and acid rain on photosynthetic parameters in rice. - Chemosphere 112: 355-361.

[29] Wang, L. Q., Yang, L. T., Guo, P., Zhou, X. X., Ye, X., Chen, E. J., Chen, L. S. (2015): Leaf cDNA-AFLP analysis reveals novel mechanisms for boron-induced alleviation of aluminum-toxicity in Citrus grandis seedlings. - Ecotoxicology and Environmental Safety 120: 349-359.

[30] Wang, Y. P., Wang, Y., Kai, W. B., Zhao, B., Chen, P., Sun, L., Ji, K., Li, Q., Dai, S. J., Sun, Y. F., Wang, Y. D., Pei, Y. L., Leng, P. (2014b): Transcriptional regulation of abscisic acid signal core components during cucumber seed germination and under $\mathrm{Cu}^{2+}$, $\mathrm{Zn}^{2+}, \mathrm{NaCl}$ and simulated acid rain stresses. - Plant Physiology and Biochemistry 76: 6776.

[31] Zheng, S., Pan, T. F., Ma, C. L., Qiu, D. L. (2017): Differential gene expression of longan under simulated acid rain stress. - Bulletin of Environmental Contamination and Toxicology 98: 726-731.

\section{APPENDIX}

Figure A1. The sequence of transcript-derived fragments (TDFs) from tomato in response to simulated acid rain

$\begin{aligned} & 1 \mathrm{M} 0 \mathrm{E} 0-1 \text { GGTCGCTGCTTGCTGATCATGTGAACACTACATTCCTGTTACCC } \\ &(175 \mathrm{bp}) \text { TCATTGACCCTACAGATATTTTGCTTCATTTGAGGTTCTCTTGC } \\ & \text { TCTCGTATGTCTCATATCATATTCCCAAAGCATCAGACCGCCTT } \\ & \text { GTTCATGGAGTTGTAAAATATTTCATCAAGCTATTGCAGATC } \\ & 2 \mathrm{M} 0 \mathrm{E} 0-2 \text { GCAGAACCTAAAATGCCTTCCATCTAGTCCTTTCAAGCAGCA } \\ &(338 \mathrm{bp}) \text { CTTGAGGAAATCGTGAAGTATAATCTGGAGAGAATTGAATAGA } \\ & \text { CGAAAACAAAGGACGAAACTATACAACTTTGGTTTATTATTCT } \\ & \text { CTGAAAGCTCTTCATATCTAACAATATCTGGAGCACAGAGTAG } \\ & \text { AAGCTATTACTATAGTTTGAATGGCAACAACGATCAGAACGAA } \\ & \text { AGTGCTTCACTATGTTCATATATTATACCCATACAGGTTGTAAT } \\ & \text { TTTCAGTTCCTAATACAGCAATATGGTGTTCCAGCATTCTTTAT } \\ & \text { AATTTGGCTAATAAATTGTCAAATTTCAACTGTTG } \\ & 3 \mathrm{M} 0 \mathrm{E} 0-3 \text { ACTCATATACGCCGGAGCAGATGGTGTTGCCGAACGCATCAAT } \\ &(164 \mathrm{bp}) \text { TGGACGTTCGTTGACTGGTTAGAACCCTGATTCAAGATACAGG } \\ & \text { TATACCAAAATATTTATGGGCTGAACTGCCTGCTACTGCTGTCT } \\ & \text { ACATGCTGAAAATTCAACCACCAATGCTGTTGT } \\ & \text { TTGGTAGATTGGTTCTCTATTGTGAGGGTGCAAATAATGTTATT } \\ & \text { TATGAGAGGTTGCGTGAAGGAGACACTGATTTGTGAAACGAC } \\ & \text { M5E0-1 } \text { TAGGACCCTCTTGGGTATTCTTGGTGGCGCTGTCCTCCAATGCC } \\ & \text { (341bp) } \text { TTTGCCACGGCGAGAGTGGTCTTAACCCTGTCACCACATAAAA } \\ & \text { ATTCCCGTCCAAAAAAAATTCACTCACTTCCCTCCATCCATTCC } \\ & \text { ATGACTTCCCCAAGGTAGCCTGGCCGGAAGTCATTTTACCACT } \\ & \text { CTCATACAACTTGCGAAGACGCCATCCAACAAGAAGCATAGCC } \\ & \text { TGAATGTTGCTCAACATCTGAACAAGCTTCTGTTGAG }\end{aligned}$


5 M5E0-2 GTCTTATAGACAATGTGGGAACTAACATTAGGAAGCCCACCAC (202bp) CGATTACACTGCTGAGGAATATGCCCATCTGTTCTCTACCAACT TGGAATCTGCATACCATCTGTGCCAACTTGCTCACCCTCTTTTG AAAGCATCTGGAAATGGATCGGTTGTCTTTATCTCTTCTGTTGC TGGTCTGGTGCACCTGTCCAGTGGTTCTATTTATGGAGCAACGA AAGGGGCAATAAATCAGCTCACGCGAAATTTGGCTTGTGAATG GGCAAAAGATAACATTCGAG

6 M5E2 AAACAGGTGAGACACGGCAACCAAAGGACTTCAACTGGTGCC (220bp) AACCGAGAACATGGCATCCCCCACTTCACAGCACATGTGAATC TACAAGCTGAGATAAATAAATTTGATTCTACTATTTTGATTTGG GTTTTCAAATTGGGCACAAATTGAAGGACAAAATCACCTCCAC CAGGAAAACCAACATATGATTGGGTATTTTTTCCTCATCAAATT ATTT

7 M6E2-1 AAAAATACAAGTAATGCATCAATAAACGCTTGATCAAAACTAG

(338bp) AATTATGTAGGTGGAATAACTATTGAAAGCTTCTTTGCAAAGT ATTCACACTAGAAACTTCAGGGTATCCATTAGATTGACATGAT GCTGAAGAAAAAAGGTGTTTCATAAGAATCCTAACTAGCAAAT GAAGCAGGCCACACATGAAAAATAAGACAAATAATACAAGTA TAATAAAAAGAACAACTGTCCGAATATTGTTTCCATATGGAGA GCACAACCATGTAAGTGTCCTAACTCTATGAAGGTTTGTCATA ATGCTGAAATTTTGAGTAGTAGTGAAGCGCATTTGCAG

8 M6E2-2 ACATCAAGAGGCGAAAAACGCCTAATTCTTCTCAGCCTTTGCC (290bp) TTCTACAGAGCAAGGTCTCGGGCCTTGTGTCGAGTTAGGAAGA TTTGGATTTGATGGAGAAGTTGATCGATTGAGGCGTGACAAGC AAGTTTTGATGACGGAACTAGTGAAGCTTAGACAGAATCAACA GAATACTCGAGCTTATCTTCGATCTTTGGAGGTAAGGTTACAA GGAACAGAGAGGAAGCAGCAACAAATGATGAACTTTTTAGCA AGAGCAATGCAAAATCCCGAGTTTGTCCAGCAG

9 M6E2-3 CTTGGAGACTTGCTTTTTCCCTGAAATTCTACCCCATTTTGAAA (264bp) GAGGAAATGTTGCATCGATGGCTTGGATTATCTCCATCGTCCTT GGTGTTGACGTGGAACAATCTCACACCACCAAGGACGATTATG GTGCTCTGGGAGCTGCTGAAAGTCGTACTAAATGGAAAGGGAC GGAGAAAAAAATGATAAAGGGACAAGTTGTCTTCTACTCTTCA TATATTCTGTGTTCACGTCGTGTATGAGAACGTTGGAGGTGAC GCAT

10 M6E2-4 TCTTGGAGCTCAATACTGCAAGGTAGTGGCAATCGTCTACCCC (212bp) AAACGATGGCCAGTCCACCAGACGATGGCCTGTCGATGGGTGG CATCGATGTGTTTTGAAGGTTGAGGAGTTTCAAGAGAAACCAA CAAACCTCATCGATGGGATGTGGTCTTACCCACGGACCGTCGA TGATGATGTCGATTGGGTTGTGATTTCTTGCAGTTTTCAG

11 MOE3 GCTCCTGGCGATGTCGAACTCGTGGCTGGTGAGTTCATGCCTTC (267bp) GGCCGGACGTGATGGCTTAGCATCATCAATTGAAAGATGGGCG ATATACTGGTTTAACGCTCTGTCTTGCCGGAGGAAAACTAGCG GGTTGCGCGTGAACGTGGAGTGTACGGCATCGCTACGTGTGAC AATCTCAGACACGGTCGCACCGAGCTCATAATCTTCTGTCGTC AATGTGGCGACTTGGATCACCACGACCTTGTCCTTCCACTGCG GGTTCTTG 
12 M1E1 (247bp)

13 M2E3 (360bp)

14 M1E0-1 (154bp)

15 M5E0

(170bp)

16 M2E2 (301bp)

$17 \mathrm{M} 3 \mathrm{E} 2$ (258bp)

18 M4E3 (211bp)

19 M5E3 (387bp)

AATATGAGAGACGATTGATACGCCTTGTTGGTAACCTCACCTC ATGACGATTATATGTGCTGGAAGATCTCATTGCTAATCAATGG GTGCGTTGCTCAACTTCGTTTTCTTTACGTACTTCAGAGTTGAC TCAAACATTATGGAATCTTGCTTCTATTTATGAGGTTTACTCAA ATCTTTACATAACCGGTCACTCCTTTCTTTACTTGATGAAATGT AAATTTGACCTTACATTATTATCTTGGTG

TTTCACTTGATGATTGGATCTTCAAATTTGGGTTATTTCCTTTAT TTTATTTTGACAATTGTTGCCGCATGTAATGGTTGGATTTTCTG GTCGCACTCCTGTGGCTTTGTCGTTATGGCTTTCCCCAAAATTC TTTTCCTTGCGGCATTTCTTCTTCTCCTTTCTTTCTGGGTTGATC TTTGTCATCAGTCAAATGATGAAGAAGATGAAGATGATGTATA TAGCCCTCGAGAAGCTTTGTTGGAGAAGAACAAGCCAAATTCA AATGCTGATAGTCGCCGAAGATGCTGCTCTTTTCGTGCTGTCAA AGTGGGAAGCCGCCAAAAAGTTGTGGTTCTGGTTACCCTGTTA GTTTTTCTG

GTGAGGACCATAATCATGAGCTTACTTGGATCCACTAGATACT GTCTATTTGAGACAAATCCTATGGGGGCATGTAGTTATGCGAC AAGGTGATTGCTTCTAAAATATTCTATTTCTACTAATGGGTAAT CTCCATTCTAAATTTCACCCAGTG

AGAAGAGATCACCAGCATGGTAAGCAACAAGTACTTTGGTAGT CTACAAGAATCGCGAGAAGCATTGCATAAATTTTAGTAACTGA GTGTCCTCTTCGTGTTTATGCAGGTGTGATCGTATACTATGGTT AGGGAAAGGTATAAAACAGATGTTTTACAAGCGCGCAGAG AACTGAGGTTTCCGTGGTGTTCTTTCTATATCCAGTAGCAAATC AATTACATTAGGGGACTCAAGACCCTGGCCAATCAGAAATAAT ACGGCAACCATGCACCGGACTTGATGCCAAAGGAAAGCACTG CCTTTGATTTTCATTATCATGAGTTGATCATCATTGTCTCTTTCA TTGAAGGGGACAATTTCAAACGATATTATGTGCCGCCGGTAGT TGTGCACATTGTCTGCATCCATTTTGCAAAAATTTCTGAAATCA TGTTCCCCTAAGAATTTCTTCGCAGCAGTTTGCATGGCTG CAGCTTGCCCCACCTCGACTATTTCACTAGGTACCTTGCTATCT CCCACCAGCATAGACCATCGGGCAACTTTGCCACTAAGGCTTA GGCAGATAAAAAAGCACCTAATGGTTTTGTCTCTGCTGGAATT TAGACCCTGATCTCTATGATTTGAACTCACTTCATTATCTAAAC CACACCCTTAGGTGCAAATACACTCCCACATATATTATCAATC AGTTATATTATCAACCAAGATTTTATCTCACCAAAAACATG TTCATCAACAATGAAGAGTTCAACAAGAAGAAAGTGATCATCA TAATGGGGGCCACACGAACGGGAAAATCCCGTCTCTCTGTTGA CTTTGCCACCCATTTTCGAGGAGAAATAATAAACTTGAACAAA ATGCAAGTTTACAAGGGACTTGAAATTGTTACAAACAAGATAA CACACACTGAGAAACAAGGTGAACGACACTATTTGTTAG GGAGTGACTCCGATGAGCCTCAAGAGTATTATACTGGCGGAGA GAAAAGCGGAATGCTTGTTCAAGATCCATCTAAGGCAAATGAT GTGAACTCAATATTCGATCAAGCTAGACAACATGCAACTGTTG AAGGACCTCCAGCATCATCTGGCTCTAGAAGCTTTATTGGAAC TGCTAGAAGACTTACGGGTGAGACTGTGTCTGCTGCTCCTCAG CCACCTGAGAGTGTTACTCATACCATCACTTTCTGGACCAATGG GTTCACTATTGATGATGGACCCTTGCGGAGGTTTGATGATCCA 
GAAAATGCCCCTTTCTTAGAGAGTATCCGGAAGTCTGAGTGCC CAAAAGAACTTGAGCCAGAAGACAGGAGGACGTCTGTCCGAG

20 M1E0-2 CAATTTTGTTGGGGAAACAAAGTCCAAGAACAATAACTTTTGG (206bp) TCACCACCAGATTTTGGGCATCTATTGCGCAATTGGACAACAT ATGATTCATCCAGAGTTAATTCTGGTTTATTATTTCCTGACTGG TTGTAGAGCCTTTGTCTGAACCTGGTACATCTTGAATTTCCAAT TGTGTGGCTTCCTGCATTACAACTTATCGGTG

21 M7E0 TGAAATGGGATTCATATCGTGTAACAAGTCGATACCAATATCA (274bp) ATTTTCCGTTCGCGAGGAATACCAGGGATATAATTAGGTAAGA CCTATAGAAATTTCCTCAATACGGGGACCGACTCAATTAGAGG AATTTCAATTTCTAAATCTTGGACTCTTACAATATGGTATAAAC ACCCTTCAGAGATCATTTTACAAGCTTTGAAATAAGAAATGAT ACGACCTCTAGGAATAGAGTTTCCCCTCTTCCACACTAAAACG GGCTCATCTGGAAAG

22 M1E2 TCCTTGCTTGAGGAATATATACTTGGGTTGATGATATACTGCTC (292bp) AATGGTGCTCATTTGTTGCACCTCTCCTTATTGGCTTCCCGATC AATAGTCAATTGTGCCCTGATGGAAGACTGGTGTCGACTATGC AATGCATCGAATTTGAATGAGTGTGAGCTTGATGGGCCACTTA TGGGTGTCTATTTTTGAATATAATTACTGCTCTTTGGTCATGAC TGATACTAATACTCCCTTTTTTTCTCTTCGGATGCCTACAATAT AAATTATGTTTCCAACTGTGTTACTTTGGT

23 M2E2 CTGCTGGACTGGATTGGAAGGAAAAAAGTGAGGATACATGAC

(281bp) TTTCATGGTTGCTTCTACTTCCTAAGTATCTCCCTTTATGGACTG ACTCCTTCACAGAACCTTGACTGAATCGACTTCTTTGTTTATCA ACCTTCTAAACTGATGGTCAAGAATCTCAACTGGTACATCCTTA TAAAAAAGCCTATCTTTCACAGCCACACTCTCTAATGGCATCAT ATAATCTGGATCACCAACACACTACTTCAAAAGTGAGATATGG AAGACCGGATGCAATGCAG

24 M7E2-1 CTTAGTCACTGGGTTTGCAAGGGATTGAAGTTCTGAGCAGTAA (298bp) GAAGCCATTGCAATTTCAGCTCCCTTGAGTCCATAATCCAAGCT TGGATTCCTTCCTGCTGTGAGATTATATGGCAACACATTGTTGT AATAGTCGTTGACAAGTTCCAAAAATTGGGCAAACATCAATTT CCCAATATATGCAAGGGCCAATCTTGTATTATCCATGGACACA CCAATAGGGGTACCTTGGAAATTTCCACCGTGTAAGGCCTTGT TTCTTGAAACATCGATCAATGGGTTGTCGTTCACTAAA

25 M7E2-2 AATTGATTGATCCTTGCTGTTAAATTCGTGGTTTTATATACAAA (310bp) TTGAGTTTGAGATGGATGTTGCATGTTCAAAAAAATTTCAATG ATCCGATGGCTTGAAGAATTGTTTTCTCGTCACTTGAGGACAA GAAATGGTTCTATGTTGAGGGTTTTGATGTGCCGTCAAATCTTG CTACATTTGATGCTTGAAAACTAATAACTGATTTCCTCATCAAG TGTTTTATGTGTATATAGTTTGTTTGGTACTTTTTTTACATGTGG ACATAAAAGAGGAATGAAAAGAAGATTGTTGATAACCAAATG CTAAA

26 M7E2-3 TCTGCAACGTCCGAGGCCTATCCGATATTGCTTTTCTGGAATGG (225bp) TCGGAATATATGTATCTATTTGGGGGCTTATTAAGTACACAAG GCACGTATCTGCTTTGCAAGTGTCCCCCGTTGCAATCGGACTTA TGGCACACAGGGGAAACACTGTACTGCTTCCTCTTTCCAGAAC 
TTCATTGAATCAAACATCGCCATTCAATAAAGAATTTTGGTGA AATCCTTC

27 M3E3 CTAATCATCTTTGGACGGTTTCTTCATCAATCTGTTGCTGAAAA (344bp) CTTTGATGGTGTTATTTTTGGGTCGTAACAATTTGTTCGGTCCC ATCCCCCAAGGAGTGGGTGAGAGGAATGATACCTTTTGGATTT GGATTTTAGCCTTTTGAACTTAATGGGAAAATCAATACAACTTT TATTATTGGAAACTCTTTCAAGGTCTTTAGCTTGATTGGCAATA AACTAACGGGGAAAGTCCCACGATCTGTGATCTTTTGTGAGTA TTTGAAACTACTTGATCTATGCAACTTTCAGTTGAATGACACGT TTCCAAACTGAATGGTATACCTATCTCAGTTGCACAGG

28 M5E3 GCCCGGGATGCATGTTCCTAGCAGGAAAGGTTGAAGAAACTCC (35bp) TCGTCCCCTGAAACATGTTATACTTGTTTCTTATGAAATCATTC ATAAAAAGGATTCTGAAGCTATTCAGAGGATCAAGCAAAAGG TATTGCTCATTGCCCTGGTGGTTGTGCTCTGTACATACCTATTT GTTCCATGTTCTAATTTTAGGTCCTCTTGAAAGTTGCCATTTTC AAAAATAAAATAAAATTTAAGTCCTCTTGAATTTTTTTTGCCCT AATAATTTGCTTCATCATGCTTATACCTGAAAGATTTTGCTTGT ATGGTCATTTGACGGTTGTTCAGTTTGGAG

29 M6E3 AGGAGAAGGGTGAGCCCTGGAGGATGATCTGGACAACAAAAA (169bp) TCCCCTCCACCGTGAATTACTTCGATGGCTTTTGGAAAGGAGG CAGTCTTGCCACATGAAAACTTGAACAAGAAGAAATCTTATCT ACATTCTAGATGCTTTTTATGTAAAAAACGGGTGATAACCG

30 M7E3-1 CATCATTGCTCTTGGTTTTGTAATCTGCCATACACAATATTCTT

(365bp) GACGATTTACTGCTAAATTTTTATGCACCGCGACTCCATCATGA ATTCTGTATGAGAAACTTCCTGGCGTACTTTCTAATATCTTAGT ACACATATCCTTTCCCAAACATTCCCTCCTTGGCCTCCTCAAAC TCACCTTCACCGGTGTATTTGCCTAGTGGCCCAATATAGTTGGC ACCTGCTCAAGTAAAACAAGCTTTCTGAGCTGCCTCAAAATTTT CTGGTCTTCCACCCCATGTCTTGAGACATGTGTTCTGAAAGCCT CTTGGATATGAAAACGATACATGTCAGGGGGTGAGAGATTGGT TCTTCGAGTTCAGT

31 M7E3-2 AAGCAACGATAAAGTGTTCTCGTTTATAAACCCTTTGTTGGTTC (193bp) ATGACGCTATCCTGCCCGTGAGGACAGATTGGAACCCTTCCTC TCCTACATGGAAAAGTATCACTCCCTAACCATGCGACGTCAAT GAAACCTTTCTTCCTTATCTGCAAATTCTTCCCCCTTGTCCAAC AACATCACTAGGTGCCAAG 